\title{
Atmospheric Pressure Low Current Plasma for Syngas Production from Alcohol
}

\author{
Ahmed Khacef, Khadija Arabi, Olivier Aubry, and Jean Marie Cormier
}

\begin{abstract}
Atmospheric pressure low current arc discharge between graphite electrodes with conical geometry in liquid ethanol/water mixture was investigated. Syngas production was demonstrated over large experimental conditions. In this paper we focus on discharge aspects. It is shown from pictures that the behavior of low current arc discharge with consumable electrodes represents non-stationary plasma.
\end{abstract}

The energetic properties of plasmas can be used to carry out many applications, particularly in discharge based systems. Recently, research interest focuses on the Non Thermal Plasma (NTP) treatment of hydrocarbons, alcohol, or biomass aimed to improve the yield of synthetic gas (syngas: $\mathrm{H}_{2}+\mathrm{CO}$ ) production at low cost $[1,4]$.

Experiments were performed on a plasma reactor consisting of two graphite electrodes with conical shape (10 mm gap). Figure 1 show the electrodes system including the position of the magnets. The magnet system produces a maximum magnetic field intensity of $140 \mathrm{mT}$ in the center of the reactor. The direct discharge was created using a sinusoidal $15 \mathrm{kV}-50 \mathrm{~Hz} \mathrm{HV}$ step-up transformer with leakage flux delivering a low current $(155 \mathrm{~mA})$. In that condition, the plasma column temperature is about $6500 \mathrm{~K}$.

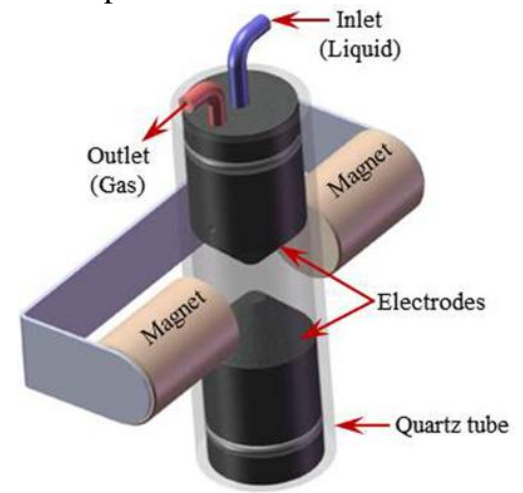

Fig. 1. Plasma reactor including the position of the magnets.

The liquid ethanol/water mixture (mole ratios from 0.1 to 0.27 ) was injected continuously at a flow rate of $75 \mathrm{~mL} / \mathrm{h}$. Crossing the electrode; the liquid was evaporated by joule effect before the discharge area. Chemical analysis shows that the electric discharge produces a hydrogen rich gas at concentrations in the range of $50 \%-72 \%$ with $\mathrm{H}_{2}$ to
$\mathrm{CO}$ ratio in the range 2-6.5. The other gas components leaving the reactor are mainly $\mathrm{CO}, \mathrm{CO}_{2}$ and $\mathrm{CH}_{4}$ at maximum concentrations of about $24 \%, 16 \%$, and $3 \%$, respectively.

It is clear from the photographs of figure 2 that the plasma discharge channel is curved. The discharge channel can be described as a plasma string with a diameter of about $2 \mathrm{~mm}$ surrounded by a larger chemical reactive region with diameter of about 5-6 mm. Its position is almost stationary. At high ethanol concentration (Fig. 2(b)), one can see formation of non conducting carbon bridges between the electrodes.

Direct discharges are usually described as stable and homogeneous plasma. However, due to the electrodes erosion, the discharge appears as a complex including the ionized medium and solid particles. Typically, spots appear on electrodes of AC discharge alternatively (Fig. 2(a) and 2(b)). These observations are correlated with voltage-current waveforms where the voltage waveform is approximately rectangular with high peaks appearing during the current reversal. Increase of electrodes erosion as results of spot formation leads to rising carbon particles density is the discharge zone. Ionic impacts at the cathode under high electric field strength are assumed to be responsible of the particles jet observed. The velocity of the ejected particles is about $10 \mathrm{~m} / \mathrm{s}$ and the phenomenon seems to appear during only a period of time lower than $2 \mathrm{~ms}$. These phenomena commonly observed during the development of high current arc seem surprising in this low current arc experiments.

In order to homogenize the electrode erosion, a sweeping effect of the discharge can be obtained by applying a constant external magnetic field. Figure 3 shows the behavior of the ionized column in the event of a discharge in (a) water vapor and (b) ethanol/water mixture when a transversal magnetic field was applied. From figure 3(b) we observe that arc spots move by jumping along the cathode with velocity of about $0.5 \mathrm{~m} / \mathrm{s}$.

To have a better understanding of processes leading to plasma erosion of electrodes, including the physical and chemical transformation in electrodes, it is important to analyze the ejected particles and electrodes after treatment. Electrodes of different materials will be studied. 

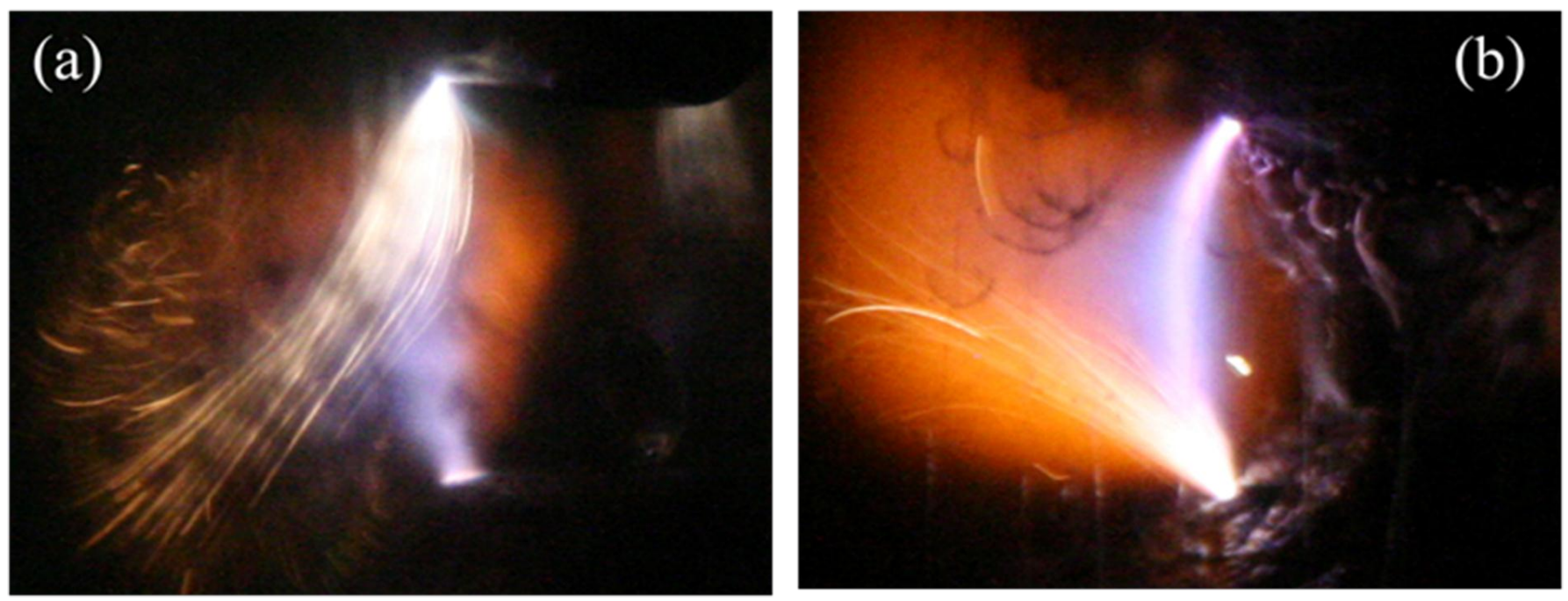

Fig. 2. Photographs of plasma column between two graphite electrodes in ethanol water mixture at atmospheric pressure (10 mm gap, 2 ms exposure time):

(a) At the beginning of the experiment. (Electrode at the bottom operated as anode)

(b) After 30 minutes of operation. (Electrode at the bottom operated as cathode)
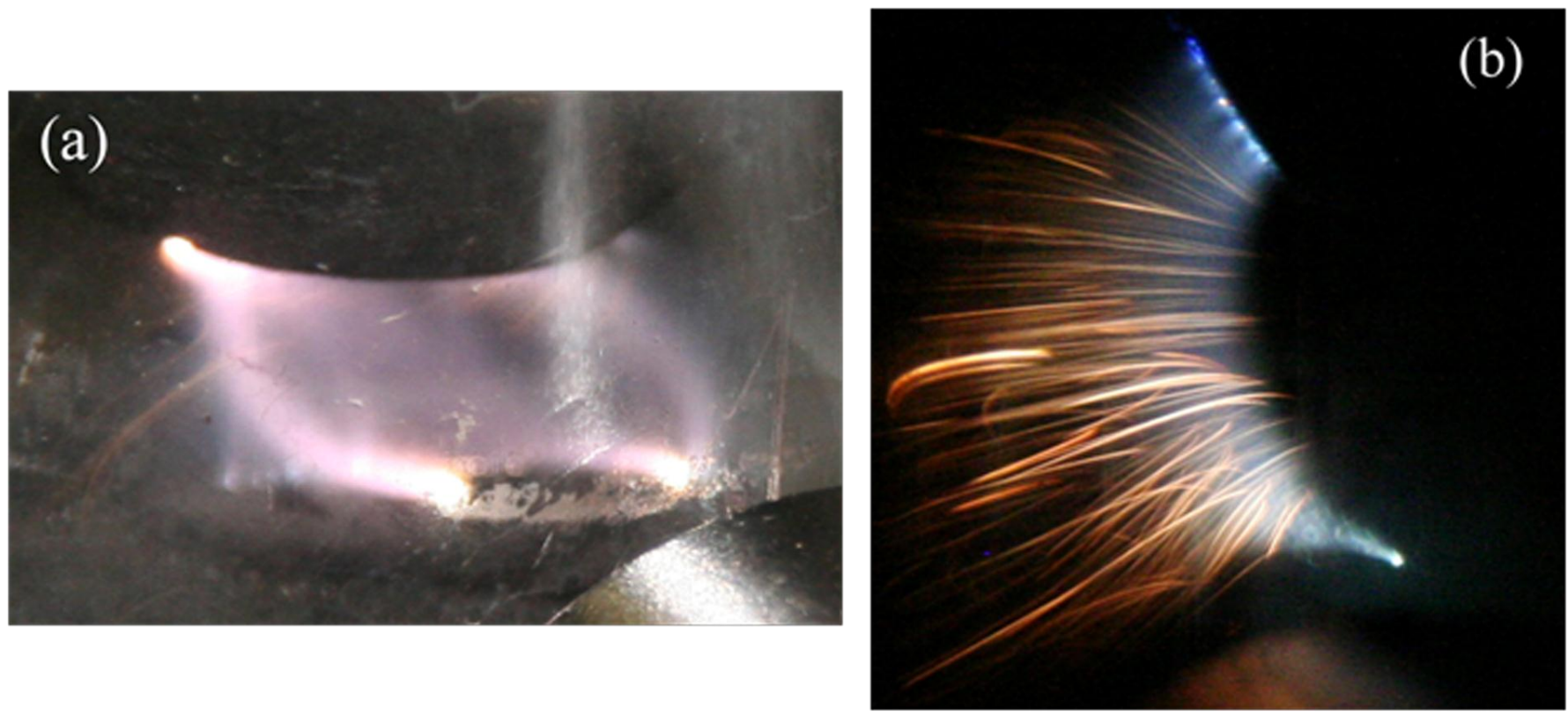

Fig. 3. Photographs of plasma column between two graphite electrodes in the presence of transverse magnetic field:

(a) in water vapor (40 ms exposure time), and (b) in ethanol $(10 \mathrm{~mL}) /$ water $(40 \mathrm{~mL})$ mixture (2 ms exposure time).

\section{REFERENCES}

[1] El Ahmar E., Met C., Aubry O., Khacef A., Cormier J.M., "Hydrogen enrichment of a methane-air mixture by atmospheric pressure plasma for vehicle applications", Chem. Eng. J., 116, 13-18 (2006).

[2] O. Aubry, C. Met, A. Khacef, J.M. Cormier, "On the use of a non-thermal plasma reactor for ethanol steam reforming", Chem. Eng. J. 106, 241-247 (2005).

[3] G. Petitpas, J.-D. Rollier, A. Darmon, J. Gonzalez-Aguilar, R. Metkemeijer, L. Fulcheri, "A comparative study of non-thermal plasma assisted reforming technologies", Intern. J. of Hydrogen Energy 32, 2848-2867 (2007).

[4] B. Sarmiento, J. Javier Brey, I. G. Viera, A. R. Gonzalez-Elipe, J. Cotrino, V. J. Rico, "Hydrogen production by reforming of hydrocarbons and alcohols in a dielectric barrier discharge", J. of Power Sources 169, 140-143 (2007). 